\title{
Discharge Model for the Lithium Iron-Phosphate Electrode
}

\author{
Venkat Srinivasan $^{*, 1}$ and John Newman ${ }^{\sharp}$ \\ Energy and Environmental Technologies Division, \\ Lawrence Berkeley National Laboratory \\ Berkeley, CA-94720
}

Submitted as a technical paper to The Journal of the Electrochemical Society

Original Submission: December 4, 2003

Revised Submission: February 28, 2004

\footnotetext{
* Electrochemical Society Active Member

${ }^{1}$ Author to whom correspondence should be addressed. e-mail: vsrinivasan@lbl.gov

${ }^{¥}$ Electrochemical Society Fellow
} 


\begin{abstract}
This paper develops a mathematical model for lithium intercalation and phase change in an iron phosphate-based lithium-ion cell in order to understand the cause for the low power capability of the material. The juxtaposition of the two phases is assumed to be in the form of a shrinking core, where a shell of one phase covers a core of the second phase. Diffusion of lithium through the shell and the movement of the phase interface are described and incorporated into a porous electrode model consisting of two different particle sizes. Open-circuit measurements are used to estimate the composition ranges of the single-phase region. Model-experimental comparisons under constant current show that ohmic drops in the matrix phase, contact resistances between the current collector and the porous matrix, and transport limitations in the iron phosphate particle limit the power capability of the cells. Various design options, consisting of decreasing the ohmic drops, using smaller particles, and substituting the liquid electrolyte by a gel are explored, and their relative importance discussed. The model developed in this paper can be used as a means of optimizing the cell design to suit a particular application.
\end{abstract}




\section{Introduction}

Since the demonstration of lithium iron phosphate $\left(\mathrm{LiFePO}_{4}\right)$ as a potential candidate for lithium insertion by Padhi et al., ${ }^{1}$ and Goodenough et al., ${ }^{2}$ considerable interest has been generated because of the numerous attractive features of the material. Not only is the material relatively inexpensive and available in nature, but it is also less toxic compared to cobalt, nickel, or manganese. ${ }^{3}$ In addition, the operating voltage of the electrode (about $3.4 \mathrm{~V} v$. Li) is ideal to maximize energy while minimizing side reactions due to electrolyte decomposition. ${ }^{3}$ Finally, the high theoretical specific energy $(\sim 580 \mathrm{Wh} / \mathrm{kg})$ makes it a very attractive candidature as a positive electrode in cells. ${ }^{3}$

However, these positive aspects are counteracted by the low electronic conductivity of the material (about $10^{-9}$ to $10^{-10} \mathrm{~S} / \mathrm{cm}$ ), ${ }^{4}$ which is thought to result in considerable ohmic drops within the electrode. In addition, it has been noted that the electrode displays limited high-rate capability, with considerable loss in utilization with increase in current, suggesting transport limitations. Various ideas have been proposed and tested to alleviate these two problems, with the most successful being the reduction of the particle size to reduce transport losses and the coating of the particles with a layer of carbon to improve the conductivity of the solid phase. ${ }^{5,6}$ Recently, efforts to introduce dopant ions into the lattice have been shown to be very successful in improving the conductivity, and consequently the performance, of the material. ${ }^{4}$

While these efforts have resulted in considerable improvement in performance, a systematic study to identify the cause for the performance limitations of the system has not been undertaken. Such an effort would be useful in both identifying the controlling mechanisms and in suggesting design changes that would improve the performance of the material. With this in 
mind, this paper aims to gain such an understanding by combining a theoretical model for the electrode with controlled experiments on well characterized cells.

Numerous models exist in the literature that use a macrohomogenous approach to describe the processes in Li-ion cells. These models have been used to understand the electrochemical characteristics of the system and in cell design. ${ }^{7-13}$ In addition, various features, such as side reactions ${ }^{14}$ and capacity-limiting mechanisms, ${ }^{15}$ have been included in order to gauge their impact on performance. Models have been developed for many different chemistries, both for the positive and the negative electrode. Except for the lithium metal negative electrode, all other chemistries share a common modeling framework that involves transport of charge across both the electronic and ionic phases in a porous electrode, transport of mass in the ionic phase, reaction at the electrode/electrolyte interface, and transport of Li ions in the solid particles. The unique features of each chemistry are then accounted for by changing the parameters that describe these processes (e.g., thermodynamic potential and diffusion coefficient) appropriately. However, the $\mathrm{LiFePO}_{4}$ electrode differs from these systems in that it undergoes a phase change with the lithiated and unlithiated forms having distinct phases, as evidenced from XRD patterns of the material at various stages of lithiation. ${ }^{1,16}$

In this paper, we develop a model that accounts for the phase change in the $\mathrm{LiFePO}_{4}$ active material in addition to describing the porous nature of the electrode. The phase change is modeled using the 'shrinking core' approach that envisions the existence of a core of one phase covered with a shell of the second phase with transport of Li-ions in the shell driving the movement of the phase boundary. While a previous study ${ }^{17}$ has incorporated this phenomenon into a battery model, they make the restrictive assumption that the system is under a pseudo steady-state, thereby limiting its applicability. This is therefore the first paper that incorporates 
the phenomenon into a porous electrode model in its complete form. In order to identify the concentration ranges that define the phase boundary, we performed experiments to measure the equilibrium potential during lithiation. Subsequently, we used galavanostatic discharge experiments at various currents to fit the model to the experimental data in order to understand the contribution of various factors that limit the performance of the electrode and suggest ways to change the design. The model developed in this paper is a precursor to the subsequent paper where, for a natural graphite/ $\mathrm{LiFePO}_{4} \mathrm{Li}$-ion cell, we optimize the porosity, thickness and capacity ratio in order to maximize the energy density for various discharge times. ${ }^{18}$

\section{Experimental}

The positive electrodes used in this study were supplied by Hydroquébec (IREQ) and consist of carbon-coated $\mathrm{LiFePO}_{4}$ electrodes made by the University de Montreal. The active mass is mixed with $4 \%$ carbon black, $4 \%$ graphite, and 10\% PVDF binder and pasted on a carbon-coated aluminum current collector. The electrode thickness was estimated to be $62 \mu \mathrm{m}$ and the mass of active material as about $7.6 \mathrm{mg} / \mathrm{cm}^{2}$. The theoretical capacity was calculated to be $1.29 \mathrm{mAh} / \mathrm{cm}^{2}$ (assuming $170 \mathrm{mAh} / \mathrm{g}$ ). After drying under vacuum at $120^{\circ} \mathrm{C}$ for 12 hours, the electrodes were assembled in a Swagelok cell in an Ar atmosphere glove box along with two layers of Celgard separator, each of thickness $25 \mu \mathrm{m}$, and a Li foil counter and reference electrodes. The reference electrode was placed to the side of the working electrode between the two layers of separator. All potentials reported in this paper are that of the positive electrode with respect to the Li reference. The electrode area was approximately $1 \mathrm{~cm}^{2}$. The cell was then filled with $1 \mathrm{M}$ lithium hexafluorophosphate $\left(\mathrm{LiPF}_{6}\right)$ in 1:1 ethylene carbonate $(\mathrm{EC})$ : diethyl carbonate (DEC) and sealed before removing from the glove box. The electrodes were then 
formed using a slow rate (about $\mathrm{C} / 25$ ) charge-discharge for five cycles in order to reach a stable capacity, after which the experiments of interest were performed. All subsequent cells were charged at a constant current of $51.7 \mu \mathrm{A} / \mathrm{cm}^{2}$ to $4.0 \mathrm{~V}$ after which the cell was held at a constant potential for 10 hours or until the current was smaller than $1 \mu \mathrm{A} / \mathrm{cm}^{2}$, so that all non uniformities in the porous electrodes were allowed to equilibrate. The cell was then rested for 1 hour and subsequently discharges were performed at various rates until a cut-off potential of $2.5 \mathrm{~V}$. With this charging algorithm, a capacity of $156 \mathrm{mAh} / \mathrm{g}$ was estimated and was taken to be the maximum capacity of the electrode $\left(\mathrm{Q}_{\max }\right)$.

In order to estimate the equilibrium potential of the material, a fully charged electrode was discharged for a known amount of time using a current of $51.7 \mu \mathrm{A} / \mathrm{cm}^{2}$, and the cell set to open circuit for as much as 48 hours. The potential of the cell after this time was seen to be invariant and was taken to be the equilibrium potential at that state-of-charge (SOC), where the SOC is defined as the current times the time divided by the maximum capacity of the cell $\left(\mathrm{It} / \mathrm{Q}_{\max }\right)$. The capacity of the cell estimated from the various constant-current periods from this experiment was estimated to be $155 \mathrm{mAh} / \mathrm{g}$, suggesting that there was little self-discharge during the experiment.

\section{Model Development}

The schematic of the cell modeled in this study is shown in Figure 1. The cell consists of a $\mathrm{Li}$ foil negative and a $\mathrm{LiFePO}_{4}$ porous positive with a porous separator between the two. As noted in the Experimental section, the positive electrode is coated on a carbon-coated current collector. The positive electrode consists of a number of particles, taken to be spherical in this 
study, with a carbon coating on the surface. This coating is considered to be thin and porous such that the lithium ion from the solution penetrates to reach the surface of the $\mathrm{LiFePO}_{4}$ particle without any resistance. The mathematical equations that describe the processes in the porous electrode are the same as those described previously ${ }^{19}$ and will not be repeated here. Briefly, these consist of a mass balance (Equation 12 in reference 19) and a modified Ohm's law in the electrolyte phase (Equation 3 in reference 19), Ohm's law in the solid phase (Equation 4 in reference 19), the Butler-Volmer equation (Equation 19 and 24 in reference 19), and a charge balance relating the reaction current to the solution current (Equation 28 in reference 19). The Butler-Volmer equation for the system requires knowledge of the surface concentration of lithium in the solid $\mathrm{LiFePO}_{4}$ particles. Previous models of lithium intercalation electrodes obtain this quantity by performing a mass balance in the solid phase by solving for the diffusion equation (see Section 3.4 in reference 19). However, as the $\mathrm{LiFePO}_{4}$ electrode undergoes a phase change, this aspect needs to be revisited.

In developing a physical picture of the phenomenon occurring in the solid-phase, two pieces of evidence need to be accounted for, namely (i) the active material has two phases; and (ii) there is bulk utilization of the material (i.e., the reaction is not just a surface phenomenon). Other electrochemical systems that also have the same two characteristics can be broadly classified into those that undergo dissolution and precipitation and those that have a shrinking core. The lead-acid battery is an example of the former. Here one of the phases $\left(\mathrm{PbSO}_{4}\right)$ dissolves into the electrolyte to form $\mathrm{Pb}^{2+}$ and $\mathrm{SO}_{4}{ }^{2-}$ ions. The $\mathrm{Pb}^{2+}$ then transports to the electrode/electrolyte interface, where reaction occurs, and the resulting product $(\mathrm{Pb})$, which is the second phase, is deposited. ${ }^{20}$ As dissolution proceeds, new sites are exposed for further dissolution. In this system, transport limitations for the dissolved species and/or its low 
solubility in the electrolyte could be a cause for the drop in utilization with current on charging. ${ }^{20}$ At present, no evidence supports such a phenomenon in the $\mathrm{LiFePO}_{4}$ system, with little dissolution of the active material expected in the electrolyte used. We therefore turn to the latter, namely the shrinking core, to describe the system.

Numerous systems which exhibit these two characteristics have been described successfully using the shrinking core approach. Two electrochemical examples include the metal-hydride electrode ${ }^{21-24}$ (used in Ni-MH cells) and the lithium-aluminum electrode (used in LiAl-FeS cells). ${ }^{17}$ Figure 2 sketches the processes that characterize this approach, where the discharging of a single particle of $\mathrm{LiFePO}_{4}$ is described. As current is passed, an electrochemical reaction occurs at the surface of the particle and can be represented as

$$
\mathrm{FePO}_{4}+\mathrm{Li}^{+}+\mathrm{e}^{-} \underset{\text { charge }}{\stackrel{\text { discharge }}{\longleftrightarrow}} \mathrm{LiFePO}_{4}
$$

In order for this reaction to continue at the particle surface, $\mathrm{Li}$ from the surface of the particle is transported to the interior through the solid lattice, similar to the processes in an intercalation electrode. After a small fraction of the Li intercalates into the lattice, denoted by y in Figure 2, further insertion results in phase separation with the formation of a new Li-rich phase having a $\mathrm{Li}$ content denoted by (1-x), where $\mathrm{x}$ can be expected to be close to zero. As the lithiation reaction occurs at the particle surface, the region adjacent to it would have a larger concentration of $\mathrm{Li}$, and hence the new phase forms as a shell along the outer circumference. This shell of Li-rich layer then covers a core of the Li-deficient layer. As the electrode is discharged further, more Li is inserted into the lattice, and this $\mathrm{Li}$ is transported to the Li-deficient core, through the shell region. This then leads to further phase separation at the phase interface, and the Li-deficient core shrinks at the expense of the Li-rich shell, which then results in the shrinking core 
phenomenon. As discharge continues, the core is completely consumed, and the whole particle is now made of the single Li-rich phase. Further discharging results in the complete intercalation of the Li into the lattice and the conversion of the particle to the fully lithiated form. This latter region is similar to the initial stages of the discharge in that the behavior is comparable to an intercalation electrode. Therefore, in summary, the shrinking core model argues that there would be a single-phase region at the beginning of discharge, followed by a two-phase region where diffusion of Li occurs through the shell with the interface moving, followed by a single-phase region in the end.

When the completely discharged electrode is charged, the process described above is repeated with a Li-rich core covered by a Li-deficient shell, with the core shrinking. In this case, the diffusion of Li occurs through the Li-deficient region, as opposed to the discharge, where it occurs through the Li-rich region. In addition, the electrochemical reaction at the interface occurs on a different material. Therefore, one can expect to see transport and kinetic parameters governing the process during charge that are different from those on discharge. For example, in the LiAl system, the diffusion coefficient in the $\beta$ phase is two-orders of magnitude larger than the corresponding value in the $\alpha$ phase. $^{25}$ In addition, the juxtaposition of the two phases is different at the same SOC. For example, in an electrode charged from the discharged state to $50 \%$ SOC, the core consists of the Li-rich phase, while for an electrode discharged to this point from a charged state, the core is the Li-deficient phase. In other words, the cycling history of the material is important in quantifying its state.

A mathematical description of the above phenomenon involves solving for the transport of Li ions in the shell. Neglecting any migration effects, and assuming a concentration- 
independent diffusion coefficient the governing equation for this process can be written in spherical coordinates as

$$
\frac{\partial \mathrm{c}_{\mathrm{s}}}{\partial \mathrm{t}}=\mathrm{D}_{\mathrm{Li}} \frac{\partial^{2} \mathrm{c}_{\mathrm{s}}}{\partial \mathrm{r}^{2}}+\frac{2 \mathrm{D}_{\mathrm{Li}}}{\mathrm{r}} \frac{\partial \mathrm{c}_{\mathrm{s}}}{\partial \mathrm{r}}
$$

The neglect of migration typically means that the transference number of electrons is assumed to be 1.0. However, $\mathrm{LiFePO}_{4}$ is an insulator, and hence this assumption is probably not justified. Instead, one could consider the particle to consist of a dilute binary electrolyte with the charge carriers being $\mathrm{Li}^{+}$and $\mathrm{e}^{-}$. Using binary electrolyte theory, one can eliminate the migration effect and describe transport using Fick's law with the diffusion coefficient modified and dependent on the relative mobilities of the two ions, as described by Newman. ${ }^{26}$ While this allows us to describe both transport processes accurately by using the diffusion equation, we are still neglecting the ohmic drop within the particle. Although this can be estimated after solving the equations, and then incorporated into the porous electrode model, the assumption that each particle is covered by a layer of carbon allows us to neglect this ohmic drop. As the conductivity of this carbon coating would be much larger than the iron-phosphate particle (The conductivity of $\mathrm{LiFePO}_{4}$ is about $10^{-10} \mathrm{~S} / \mathrm{cm}$; that for graphite is $300 \mathrm{~S} / \mathrm{cm}$.), the electron is envisioned to travel over the circumference to reach the reaction site, rather than travel within the particle. Therefore, no ohmic drop should exist within the particle. However, one would expect to have an ohmic drop due to the transfer of the electron from the current collector through the porous electrode. This is accounted for in the model using Ohm's law in the matrix phase.

By neglecting the initial single-phase region and assuming that the interface between the two phases is at equilibrium, Equation 2 is solved with the initial and boundary conditions, 


$$
\begin{array}{ll}
\mathrm{c}_{\mathrm{s}}=\mathrm{c}_{\mathrm{o}} & \text { at } \mathrm{t}=0 \\
\mathrm{D}_{\mathrm{Li}} \frac{\partial \mathrm{c}_{\mathrm{s}}}{\partial \mathrm{r}}=\frac{\mathrm{i}_{\mathrm{n}}}{\mathrm{F}} & \text { at } \mathrm{r}=\mathrm{r}_{\mathrm{o}} \\
\mathrm{c}_{\mathrm{s}}=\mathrm{c}_{\mathrm{eq}} & \text { at } \mathrm{r}=\mathrm{r}_{\mathrm{i}}(\mathrm{t})
\end{array}
$$

where $c_{o}$ is the concentration of the Li-deficient phase where phase separation occurs, $c_{\text {eq }}$ the concentration of the lithium-rich phase, assumed to be in equilibrium with the Li-deficient phase, $r_{i}$ the position of the interface, which is a function of the time of discharge, and $i_{n}$ the reaction current at the particle surface. Neglect of the initial single-phase region is justified based on the narrow composition range of this region (see Figure 3 and the explanation in the text). In addition, the model ignores any volume change occurring from the insertion of lithium into the lattice in order to keep the analysis simple. Padhi et al. show that the lattice undergoes a $6.81 \%$ increase in volume on lithiation. ${ }^{1}$

In order to solve this system of equations, the unknown interface position needs to be evaluated. This is done by developing a mass balance at the interface, written as

$$
\left(c_{o}-c_{e q}\right) \frac{d r_{i}}{d t}=\left.D_{L i} \frac{\partial c_{s}}{\partial r}\right|_{r=r_{i}}
$$

As written, the equations represented an electrode discharged from a completely charged state. Simulations from partial charge (or discharge) can be easily incorporated into this framework by first calculating the radius of the shell using a mass balance and subsequently solving for diffusion and phase movement as detailed above.

The end-of-discharge occurs when the surface concentration of lithium reaches a predetermined maximum concentration (on discharge), calculated using faradays law and the maximum capacity of the electrode $\left(\mathrm{Q}_{\max }\right)$ and assuming a density of $3.6 \mathrm{~g} / \mathrm{cc}$ for the material. 
Depending on the current, diffusion coefficient, particle size, and the concentration range of the single-phase region, this can occur after the core is consumed, or much before (see Appendix A). If the core is consumed, then the whole electrode is uniformly in one phase and behaves like an intercalation electrode. Under these conditions the boundary condition at $r=r_{i}(t)$ is substituted by

$$
\mathrm{D}_{\mathrm{Li}} \frac{\partial \mathrm{c}_{\mathrm{s}}}{\partial \mathrm{r}}=0 \quad \text { at } \mathrm{r}=0
$$

and the equations solved.

Equations 2 to 5 constitute the set of equations needed to account for transport in the solid phase. This problem is solved numerically for a constant current density and incorporated into the porous electrode model using a pseudo two-dimensional approach, as detailed below.

\section{Solution Procedure}

Equations 2 to 4 constitute a moving-boundary problem that was solved by making the coordinates stationary by substituting the transformation

$$
x=\frac{r-r_{i}}{r_{o}-r_{i}}
$$

into Equations 2 to 4, resulting in

$$
\begin{aligned}
& {\left[r_{i}+x\left(r_{o}-r_{i}\right)\right](x-1)\left(r_{o}-r_{i}\right) \frac{d r_{i}}{d t} \frac{\partial c_{s}}{\partial x}+\left[r_{i}+x\left(r_{o}-r_{i}\right)\right]\left(r_{o}-r_{i}\right)^{2} \frac{\partial c_{s}}{\partial t}=} \\
& D_{L i}\left[r_{i}+x\left(r_{o}-r_{i}\right)\right] \frac{\partial^{2} c_{s}}{\partial x^{2}}+2 D_{L i}\left(r_{o}-r_{i}\right) \frac{\partial c_{s}}{\partial x}
\end{aligned}
$$




$$
\begin{array}{ll}
\mathrm{c}_{\mathrm{s}}=\mathrm{c}_{\mathrm{o}} & \text { at } \mathrm{t}=0 \\
\mathrm{c}_{\mathrm{s}}=\mathrm{c}_{\mathrm{eq}} & \text { at } \mathrm{x}=0 \\
\mathrm{D}_{\mathrm{Li}} \frac{\partial \mathrm{c}_{\mathrm{s}}}{\partial \mathrm{x}}=\left(\mathrm{r}_{\mathrm{o}}-\mathrm{r}_{\mathrm{i}}\right) \frac{\mathrm{i}_{\mathrm{n}}}{\mathrm{F}} & \text { at } \mathrm{x}=1 \\
\left(\mathrm{c}_{\mathrm{o}}-\mathrm{c}_{\mathrm{eq}}\right)\left(\mathrm{r}_{\mathrm{o}}-\mathrm{r}_{\mathrm{i}}\right) \frac{\mathrm{dr}}{\mathrm{dt}}=\left.\mathrm{D}_{\mathrm{Li}} \frac{\partial \mathrm{c}_{\mathrm{s}}}{\partial \mathrm{x}}\right|_{\mathrm{x}=0}
\end{array}
$$

A new governing equation

$$
\frac{\mathrm{dr}}{\mathrm{dx}}=0
$$

is now introduced, allowing us to treat the position of the phase-interface as a second unknown with Equation 9 serving as the boundary condition. This simultaneous approach is similar to that used by Newman to solve for the eiganvalues of the Graetz problem ${ }^{27}$ and by White et al. to solve for the position of the reaction plane during copper deposition. ${ }^{28}$ This resulted in a set of two partial differential equations, which were discretized using finite differences, and solved for a constant current using the Band subroutine, developed by Newman. ${ }^{29}$ In the case where this model is incorporated into the porous electrode model, a pseudo 2-D approach was used, where one Band subroutine was used for the porous electrode dimension and a second Band subroutine was used in the particle dimension. This is similar to the approach used in previous studies. ${ }^{30}$ Initial guesses were provided for all quantities in the electrode dimension, including the reaction current (the current produced due to electrochemical reaction normal to the active material surface). The reaction current was used as input into the particle model, which was used to calculate the surface concentration. This surface concentration was fed back to the electrodescale model, and iteration commenced. This procedure was continued until convergence was reached. Time stepping was accomplished using the Crank-Nicolson method. Previous models 
for Li-ion cells with intercalation electrodes have used, for example, the Duhamel superposition integral to describe analytically the time-dependent flux at the particle surface. ${ }^{8}$ Such an analytic approach is not possible in this system because of the non linearity introduced by the moving phase boundary. The boundary condition change from the shrinking core electrode to the intercalation electrode was performed by assuming the core is consumed when the radius of the core reaches $1 \%$ of the radius of the particle. Simulations using values of $0.1 \%$ and $10 \%$ showed little difference as the amount of active material at the center of the core is extremely small owing to the small volume, and hence $1 \%$ was used. Appendix A illustrates the change in boundary conditions visually.

\section{Results and Discussion}

\section{Equilibrium Potential Measurements:}

Figure 3 plots the equilibrium potential measured as a function of the state-of-discharge (SOD, defined as 1-SOC) for the $\mathrm{LiFePO}_{4}$ electrode. The experiment was performed on two different cells in order to ensure consistency and Figure 3 shows the results from one of these cells. The points were generated by discharging the cell to a specific SOD and then resting (zero current density) until a steady state voltage was reached. A plot of cell voltage $v s$. log (time) was used to ensure that the voltage was indeed at a steady state. Points taken at closed-circuit potentials above $3.5 \mathrm{~V}$ vs. Li resulted in the potential decreasing to this point at the end of the rest period. This suggests that there may be a side reaction occurring at higher potentials. However this side reaction did not seem to result in any capacity fade (as noted in the Experimental section), and any error in the estimation of the SOD is expected to be small. No further effort was made to quantify this decrease in potential. 
Figure 3 shows an initial narrow region where the potential changes with lithation, followed by a potential-invariant region and finally a steep decrease in potential as the sites are filled with Li. By the Gibbs phase rule, a single-phase region would be characterized by a changing potential with concentration, while a two-phase region would have a potential that is invariant, and therefore Figure 3 provides a means of identify the concentration ranges that define the phase boundaries in the shrinking core model. The concentration ranges of the two single-phase regions were estimated from the point where the potentials deviate from the invariant region, corresponding to a SOD of 0.02 and 0.9525 for the Li-deficient and Li-rich phases, respectively. For comparison, in the LiAl system, Wen et al. estimate 9.2 atomic percent (a/o) Li for both the $\alpha-\mathrm{Al}$ and $\beta-\mathrm{Al}$ phases. ${ }^{31}$

It should be noted that the existence of the single-phase regions has not been reported previously in the literature for this material. Yamada et al. performed XRD analysis on chemically lithiated $\mathrm{LiFePO}_{4}$ electrodes and were able to separate the two phases based on the evolution of the lattice constants in the unit cell. ${ }^{16}$ They conclude that the electrode has two phases at all SOC. However, the authors conducted experiments in samples with significant differences in the lithiation (approximately 20\%) and hence did not access the ranges reported in Figure 3. We suggest that experiments conducted with lithiation such that the electrode remains in the narrow single-phase regions may help in confirming the existence and the composition range of these regions. While open-circuit experiments can be misinterpreted because the resting time was not long enough for a true steady state to be reached, a spectroscopic investigation would leave no doubt as to the existence (or nonexistence) of a single-phase region at a particular SOC. However, as we note in Appendix A, the concentration range of the single-phase region 
and the diffusion coefficient both affect the change in concentration across the particle with time, and hence an incorrect estimation of one would be offset by the other quantity.

While Figure 3 helps complement the shrinking core model, no information is known at this point on the actual juxtaposition of the two phases. While a shrinking core is attractive because of its extensive use in previous systems, arguments can be made that the diffusion of Li in the material may be preferential in one of the lattice dimensions as opposed to the others, leading to the phase change not being symmetric. In addition, grain-boundary diffusion may dominate the transport of $\mathrm{Li}$ into the material with the diffusion in the single-phase region restricted to a narrow distance. While the former will yield a non symmetric shrinking core, the latter would lead to one particle having multiple shrinking cores in it, similar to particles joined into an agglomerate. Modeling the former process would involve a two-dimensional model in the particle scale, while the latter would result in a three-scale model. ${ }^{32}$ With little information available, we have chosen to study the system by assuming a shrinking core. Further understanding of the phase evolution is needed in order to refine the model.

Although no systematic study was undertaken to ascertain whether a hysteresis exists in the equilibrium potential, one equilibrium experiment was performed from a fully discharged electrode which was taken to approximately $50 \%$ SOC and was found to be at the same potential as that measured when discharging a fully charged electrode to this SOC, suggesting no history dependence.

In order to use the data shown in Figure 3 in the model, curve fitting was used to determine the equilibrium-potential expression. As mentioned previously, the particle model neglects the initial single-phase region, and the process is assumed to start with the particle 
having a shell-core configuration. Therefore, the following equation was fit to the data in Figure 3 , where the steep decrease in potential at low SOD was neglected

$$
\begin{aligned}
\mathrm{U}= & 3.114559+4.438792 \tan ^{-1}\left(-71.7352 \frac{\mathrm{c}_{\mathrm{s}}}{\mathrm{c}_{\max }}+70.85337\right) \\
& -4.240252 \tan ^{-1}\left(-68.5605 \frac{\mathrm{c}_{\mathrm{s}}}{\mathrm{c}_{\max }}+67.730082\right)
\end{aligned}
$$

where $\mathrm{c}_{\max }$ is the maximum concentration of Li that can be incorporated into the lattice (taken to be $0.02095 \mathrm{~mol} / \mathrm{cm}^{3}$ ). Figure 3 also shows the fits of this equation to the experimental data.

\section{Model-Experimental Comparisons:}

The equilibrium-potential expression, given above, along with the concentration ranges was used in the porous electrode model to generate discharge curves as a function of discharge rate. The transport properties for the electrolyte were taken from literature values for the salt in comparable solvents, and the Bruggeman equation used to account for porosity and tortuosity. ${ }^{33}$ Appendix B lists the electrolyte parameters used in this paper while Table 1 lists the designadjustable parameters used. There have been a few measurements of the diffusion coefficient of $\mathrm{Li}$ in $\mathrm{LiFePO}_{4}\left(\mathrm{D}_{\mathrm{Li}}\right)$ in the literature, and values range from $10^{-17}$ to $10^{-22} \mathrm{~m}^{2} / \mathrm{s} .{ }^{34-36}$ Prosini et al measured this quantity using both a current pulse and an impedance technique on hydrated $\mathrm{FePO}_{4}$ and reported values ranging from $2.7 \times 10^{-21}$ to $8.1 \times 10^{-22} \mathrm{~m}^{2} / \mathrm{s}$, depending on the concentration. The authors also perform current pulse experiments on $\mathrm{LiFePO}_{4}$ prepared from a solid-state method and estimate values ranging from $1.8 \times 10^{-18}$ to $2.2 \times 10^{-20} \mathrm{~m}^{2} / \mathrm{s}$, depending on the concentration. However, both these materials show sloped equilibrium potentials, unlike that expected from a two-phase system. It is therefore possible that these values are on a material different from ones used in this paper. In contrast, Franger et al. report $\mathrm{D}_{\mathrm{Li}}$ measured using an 
impedance technique on thin-film materials that appear to be similar to ones used in this study. They conclude that the value is a constant for the whole range of composition and around $10^{-17}$ to $10^{-18} \mathrm{~m}^{2} / \mathrm{s}$. This provides us with a starting point for $\mathrm{D}_{\mathrm{Li}}$ in our simulations.

The determination of a more accurate value of $\mathrm{D}_{\mathrm{Li}}$ than the one reported by Franger et al can be problematic in these two-phase materials. For example, both Prosini et al. and Franger et al. perform experiments under conditions where semi-infinite diffusion occurs to estimate the value of the $\mathrm{D}_{\mathrm{Li}}$. If we assume that the juxtaposition of the phases is in the form of a shrinking core, then, in order for semi-infinite conditions to be achieved, the electrode has to be discharged (or charged) to a SOC such that the core is far away from the surface. If the core were close to the surface of the particle, as lithium is intercalated (or ejected), the phase-interface would also be moving and hence a moving-boundary problem would have to be solved to get the diffusion coefficient. In addition, both these techniques need the derivative of the equilibrium potential with concentration. Considering the steep change in potential in the narrow single-phase region reported in Figure 3, accurate determination of the derivative would be critical. Furthermore, the narrow single-phase region would also mean that a strong concentration dependence would make the analyses complicated.

No measurement of the kinetic parameters (exchange current density and transfer coefficient) seems to have been made in these materials, although, in general, the kinetics of Li intercalation is thought to be facile. In addition, as $\mathrm{LiFePO}_{4}$ is known to be a poor conductor, we expect ohmic drops in the matrix phase to be important. This number would probably be dependent on a number of factors, including the carbon content, the integrity of the carbon coating, and the calendaring process, and therefore, probably, electrode specific. In addition, it has been shown that the structure of the coating $\left(\mathrm{sp}^{2}-\right.$ versus $\mathrm{sp}^{3}$-coordinated carbon) can have an 
impact on the electronic properties and the performance of the materials. ${ }^{37}$ In addition, it has been noted previously that a contact resistance between the current collector and the porous electrode can have a significant impact on performance. ${ }^{38}$ Experimental studies in $\mathrm{LiFePO}_{4}$ electrodes have indicated that using a carbon-coated current collector was critical in improving performance, suggesting that this contact resistance was important. ${ }^{39}$ Like the matrix conductivity, this quantity would also be dependent on a number of factors and hence, difficult to determine. Therefore it was decided that both these quantities would be extracted by fitting the model to the experiments.

Figure 4 (a) shows experimental discharge curves taken at various rates ranging from about $\mathrm{C} / 5\left(0.26 \mathrm{~mA} / \mathrm{cm}^{2}\right)$ to $5 \mathrm{C}\left(6.5 \mathrm{~mA} / \mathrm{cm}^{2}\right)$. As noted in the experimental section, the cell was charged using a constant current followed by a constant potential, which ensures that the whole porous electrode was at the same SOD (assumed to be 0.0) before the curves were generated. The plots are shown as a function of capacity where the mass is that of the active material only (no carbon, binder etc.). All the curves show an initial steep drop in potential followed by a plateau region. We believe that this steep drop is caused by the initial single-phase region, not described in the model. The curves show three characteristics that we aim to describe using the model, namely (i) a drop in utilization with increasing current density; (ii) a decrease in the mid-plateau potential with increasing current density; and (iii) a slope in the discharge curves at higher current densities in the plateau region, with the slope increasing with current. The drop is utilization is a transport-limited process. The other two features can be caused by a combination of transport, kinetic, and/or ohmic limitations in the system. Note that the maximum capacity in these electrodes is $156 \mathrm{mAh} / \mathrm{g}$, while the discharge at $0.26 \mathrm{~mA} / \mathrm{cm}^{2}$ shows a capacity of $148 \mathrm{mAh} / \mathrm{g}$, illustrating the poor rate capability of the iron-phosphate electrode. 
In order to fit the model to the data, we first use the utilization (ratio of the actual capacity to the maximum capacity, $\mathrm{Q}_{\max }$ ) to extract the transport properties. Simulations show that the liquid phase has little impact on the voltage drops in the cell (see Figure 7), thereby suggesting that this drop in utilization is dictated by solid-phase transport. As the particle size and the concentration ranges are fixed, we are left with only one parameter that dictates the endof-discharge, namely $\mathrm{D}_{\mathrm{Li}}$. Therefore, we can use the measured utilization to extract $\mathrm{D}_{\mathrm{Li}}$ from the simulation, keeping in mind the values measured in the literature. Fixing this quantity allows us to estimate the potential drop arising from transport losses, which leaves us with the kinetics and the ohmic drop to fit the other two signatures seen in Figure 4 (a), namely the voltage drop and the slope in the voltage-capacity curve. Therefore, attempts were made first to estimate $\mathrm{D}_{\mathrm{Li}}$ by choosing a current and fitting the model to the experimental utilization, and then using this value to predict utilization at other currents.

The result of this process are shown in Figure $4(\mathrm{~b})$, where the discharge curve at a current of $1.3 \mathrm{~mA} / \mathrm{cm}^{2}$ was used as the base case to fit the end of discharge. While a contact resistance between the current collector and the porous electrode was used to generate Figure 4(b), no attempt was made to fit the voltage drops. (This issue is addressed in the next graph.) Using a diffusion coefficient of $5 \times 10^{-18} \mathrm{~m}^{2} / \mathrm{s}$ resulted in an excellent fit for the end-of-discharge at the selected current density. This value is within the range reported by Franger et al. ${ }^{36}$ However, using this value of $\mathrm{D}_{\mathrm{Li}}$ gives poor predictions at all the other current densities. Figure 4 (b) shows two of these curves to illustrate this point. At current densities higher than the ones used to extract $\mathrm{D}_{\mathrm{Li}}$, the model under predicts the utilization while at lower current densities it over predicts the utilization. This was found to be true independent of the current density used to extract $\mathrm{D}_{\mathrm{Li}}$. The simulations suggested that at each rate a different $\mathrm{D}_{\mathrm{Li}}$ was needed to fit the end 
of discharge. Such a phenomenon (a rate-dependent diffusion coefficient) has been noted previously in other mathematical models for battery electrodes. ${ }^{38,40,41}$ Two possible reasons for such a phenomenon are a diffusion coefficient that is a function of concentration, which manifests itself as a rate-dependent diffusion coefficient, ${ }^{38,40,41}$ and/or the existence of a particlesize distribution. ${ }^{42}$ We first tested the former theory by incorporating a concentration-dependent diffusion coefficient into the model. The concentration dependence was incorporated using an equation of the form

$$
D_{\mathrm{Li}}=\left(\mathrm{D}_{\mathrm{o}}-\mathrm{D}_{1}\right)\left(\frac{\mathrm{c}_{\text {max }}-\mathrm{c}}{\mathrm{c}_{\max }-\mathrm{c}_{\mathrm{eq}}}\right)^{\mathrm{n}}+\mathrm{D}_{1}
$$

While $D_{o}$ and $D_{1}$ fix the diffusion coefficient at the concentration limits, $n$ controls the change from one to the other. Several different parameter values were tested, but no one functionality was successful in predicting the utilization at all current densities. The only way for us to make the predictions needed was to expand the range of the single-phase region significantly from what we report in Figure 3. It appears that, when the concentration range is small, the effect of having a concentration-dependent diffusion coefficient is limited. On the other hand, for a wide concentration range (i.e., an intercalation electrode) this has been shown to be a significant contributor in predicting the rate dependence of the final utilization. ${ }^{41}$ We therefore abandoned this approach and concentrated on using a particle size distribution, which, as described below, gave us the prediction we seek.

The particle size used in Figure 4 (b) was based on a BET experiment conducted on this electrode which indicated a diameter of $104 \mathrm{~nm}$, assuming spherical particles and a density of 3.6 $\mathrm{g} / \mathrm{cm}^{3}$. However, Transmission Electron Microscope (TEM) imaging on this electrode suggests 
that a wide particle-size distribution actually exists, with diameters ranging from 50 to $250 \mathrm{~nm}$. As incorporating a true distribution is difficult, the model was modified to incorporate two particle sizes. The methodology is similar to that outlined by Darling and Newman and assumes that the electrode having the two particle sizes has the same surface area and the same volume fraction as the actual electrode. ${ }^{42}$ This allows us to fix the radius of the average particle, the radius of the small particle, the radius of the large particle and the average volume fraction and calculate the volume fraction of the small and large particles in the electrode. Once these are fixed, the reaction current equation is modified, as illustrated by Darling and Newman, ${ }^{42}$ in a manner that is similar to having two simultaneous reactions in the electrode with each reaction having a different surface area for reaction. Finally, the particle transport equation was solved for separately in each particle by having two different Band subroutines in this pseudo-second coordinate. This approach can be extended to include more sizes, if needed. An alternate formulation for incorporation of a particle size is to use packing theory as described by Nagarajan et al. ${ }^{43}$

The particle-size distribution introduces two new unknowns into the model, namely, the radius of the small and the radius of the large particles, in addition to the diffusion coefficient. Using the fact that the smaller particles are more important at larger current densities, while the larger particles are more important at smaller current densities, the parameters were adjusted to give the best visual fits (see Table 1 for parameter values). While the estimated diffusion coefficient is within the range estimated by Franger et al., ${ }^{36}$ the radii of the two particles are within the range expected based on the TEM images.

We are still left with estimating the kinetic and the ohmic limitations in the cell. This is accomplished using the other two signatures seen in Figure 4(a), namely the voltage drops and 
the slope in the voltage-capacity curves. The slope is caused by an increase in the polarization with SOD. Diffusion is one candidate for such a signature, where a decrease in concentration results in the overpotential increasing in order to pass the same current density, as reflected in the concentration-dependent Butler-Volmer equation. However, simulations based on the transport parameters estimated above do not give us the necessary slope. In addition, we attempted adjusting the transfer coefficient in the kinetic expression to see if the discharge curves can be made more sloped, ${ }^{44}$ but this was also not useful in achieving the needed fits. Finally, we are left with an ohmic effect, with an increasing ohmic drop with SOD. One way for this to occur is if a non uniform reaction distribution exists in the porous electrode, with the distribution changing in such a way that the current travels though the less conductive path at increasing larger distances as discharge proceeds. As the solution conductivity was large enough not to have a significant impact, we decreased the matrix conductivity and increased the exchange current density of reaction to fit the slope. A smaller exchange current density makes the reaction distribution uniform, and hence the slope becomes smaller and hence deviates from the data. A larger value than the one listed in Table 1 showed no difference in slope, and a small decrease in the voltage drop. However, these values resulted in a steep reaction distribution at short times necessitating smaller time-steps and hence the value listed in Table 1 was used in all simulations. The large value of the exchange current density also means that the value of the transfer coefficient used is immaterial, and all the simulations in this paper use a value of 0.5 .

With these parameters now fixed, the model was used to predict the voltage drop in the cell. However, the experimental data in Figure 4(a) were seen to have a larger potential drop compared to the predictions, and hence the residual resistance was accounted for using the contact resistance. The value used for this quantity $\left(0.0065 \Omega-\mathrm{m}^{2}\right)$ is comparable to values 
report by Arora et al. for a graphite/ $\mathrm{LiMn}_{2} \mathrm{O}_{4}$ system (about $0.003 \Omega-\mathrm{m}^{2}$ ). ${ }^{38}$ The resulting model-experimental comparisons are shown in Figure 5, where excellent agreement is seen.

Considering that we have adjusted six parameters, namely, the radius of the small and large particles, the diffusion coefficient, exchange current density, matrix conductivity and contact resistance, to get the model-experimental fits in Figure 5, a few words need to be said about the validity of the parameters estimated and our confidence in them. As mentioned earlier, the transport processes can be estimated without any interference from the kinetic and ohmic parameters and hence we believe that we have reasonable estimates in the three parameters, namely, the radius of the small and large particles and the diffusion coefficient, that describe this feature. While a true particle-size distribution would be desirable, having only two particle sizes offers a compromise between accurate fits and ease of implementation. The kinetic parameters are taken to be such that the drop from them is relatively small compared to the other drops in the system and hence any error in this would be negligible. It is in accessing the relative magnitude of the matrix resistance and the contact resistance that we have the greatest variability. While the numbers in Table 1 are the only combination that yielded the fits seen in Figure 5, especially in achieving the slope in the voltage-capacity curve, local effects within the particle, not included in the model, may be contributing to this feature and manifesting itself as a low matrix-phase conductivity. While the matrix conductivity used here is five orders of magnitude larger than that reported in the literature for a pure $\mathrm{LiFePO}_{4}$ electrode, our expectation would be that the carbon in the material would have enhanced electron conduction on a macroscopic scale. This suggests that phenomenon on a particle scale may be important. For example, if the particles were not completely covered with carbon, then ohmic drops within the particle could become important. In order to address this issue, we tried fitting the model to the 
experiments using a film resistance, similar to those reported previously, ${ }^{9}$ representing an ohmic drop between the active material particle and the solution. While previous studies have thought of this resistance as arising from the Solid-Electrolyte Interphase (SEI) layer covering the particles, it could also be representative of an ohmic drop arising from within the particle. However, this did not provide us with reasonable fits for the slope. A film resistance has a similar effect to a low exchange current density in that the reaction distribution in the porous electrode becomes uniform, and this uniformity destroys the increasing polarization with SOD that we seek. On the other hand, a film resistance that increases with increasing SOD could give us the required effect by providing this polarization, even with a uniform reaction distribution. However, a physical explanation for an increasing ohmic resistance within the particle is difficult to find from existing knowledge of electron conduction in $\mathrm{LiFePO}_{4}$. It has been reported that the conductivity of the material does not change with lithium content, because locally, the material has either iron in the $2+$ state (in $\mathrm{LiFePO}_{4}$ ) or in the $3+$ state (in $\mathrm{FePO}_{4}$ ), but never both, because of the two-phase nature of the material. ${ }^{4}$ In terms of Figure 2, this can be interpreted to mean that over a wide SOD range, the material has a shell at one (fixed) concentration and the core at another (fixed) concentration. Changing the SOD only changes the position of the phaseinterface and consequently the volume fraction of each phase, but not the concentrations. Hence the insertion (or extraction) of charge carriers $\left(\mathrm{Li}^{+}\right.$and $\left.\mathrm{e}^{-}\right)$results in little change in conductivity, with only the changing volume fraction being a contributor. While this is indeed the process during much of the discharge regime (except for the single-phase regions), the argument is based on a thermodynamic reasoning. During transients, as demonstrated in this paper (see Figure A1), the concentration of lithium in the particle can change in the shell. This change in concentration may result in a change in the conductivity which may, in turn, result in some of these SOD- 
dependent effects exhibited by the discharge curves. More information on electron conduction in this material would be helpful in seeing if such local effects are important and in accessing the validity of the estimated parameters. In addition, other experiments, for example, impedance and current-interrupt, and model fits to them, would help in understanding more about these features. These involve modeling the growth of new shell regions of different composition growing from the surface over the existing shell-core configuration, thereby making them complicated. ${ }^{25} \mathrm{We}$ have recently compared the model developed in this paper to six different materials with varying carbon contents, particle sizes and with or without dopant ions, from which electrodes were made of different thickness and loading. ${ }^{45}$ Reasonable predictions were seen in all six cases using the diffusion coefficient and exchange current density extracted from this paper, suggesting that the model, as described here, captures all the essential features that govern the behavior of the iron-phosphate electrode.

The increasing ohmic polarization as discharge proceeds is captured in Figure 6 (a) and (b) where the pore-wall flux of the intercalation reaction is plotted across the porous electrode during discharge. As the model has two particle sizes, the figure shows this quantity for both sizes, where the movement of the reaction from the current collector towards the separator is clearly seen. At short times, the reaction is predominantly near the current collector, and there is as much as an order of magnitude difference between the rate at this region compared to the separator end of the porous electrode. As discharge proceeds, the particles near the current collector start to lithiate more when compared to the other parts of the porous electrode. This results in a greater resistance for reaction, thereby moving the reaction into the porous electrode. Although, as discharge proceeds, the profiles become more uniform when compared to the initial times, the movement of the peak into the electrode is clearly seen. This movement into the 
electrode means that the current travels a larger distance through the matrix phase before it transfers into the solution. As the matrix conductivity is low, this leads to the increasing resistance seen as discharge proceeds. Clearly the movement of this reaction front would change if the matrix is made more conductive and can even result in the front propagating from the separator towards the current collector (for $\sigma>>\kappa$ ). Note that the sharpness of the front is governed by the exchange current density of the reaction, with the front being more pronounced with a larger value. ${ }^{25}$

Figure 6 (a) and (b) also captures the differences between the reaction rate of the large [6 a)] and small [6 (b)] particles. Although not clear from the figure, a superposition of the two plots shows that, at short times, the curves for the two sizes are the same, but as reaction proceeds, the reaction rate is higher for the larger particle compared to the smaller ones, although both show the movement of the front towards the separator. As discussed by Darling and Newman, ${ }^{42}$ at short times the radii of the particles are immaterial as the concentration profiles are confined to the surface of the particles, and hence both particles have similar values. From Faraday's law, the same reaction rate results in the same number of moles of $\mathrm{Li}$ intercalating into both particles. This leads to a great concentration change in the smaller particles owing to its smaller volume. This change in concentration then changes the potential of the system, which in turn leads to an increase in the resistance to reaction, resulting in a decrease in the reaction rate. This decrease allows the larger particles to 'catch up' to the smaller particles. As noted by Darling and Newman, ${ }^{42}$ these differences between the particles are influenced by the shape of the equilibrium potential curve. While a slopey equilibrium results in a decrease in the mismatch between the two particles, a flat potential enhances these differences. 


\section{Potential-Drops in the Cell:}

The model can now be used to access the factors that limit the performance of the material by breaking down the drops in the system into its various components. We illustrate this at a current density of $2.6 \mathrm{~mA} / \mathrm{cm}^{2}$ in Figure 7. The experimental data and the model fits represent the potential between the current collector and the reference electrode placed in the separator. This potential can be split into various components in many different ways. In Figure 7 (a) we do this by evaluating the drop through the contact resistance (denoted 'contact resistance' in the figure), followed by the drop to get the current from the current collector end of the porous electrode to the separator end through the matrix phase (denoted 'matrix resistance'), followed by the drop to transfer the current from the matrix to the solution (denoted 'overpotential drop'), and finally the drop from the separator end of the porous electrode, in the solution, to the reference electrode. These drops are subtracted sequentially from the equilibrium potential and marked in the figure, where the drop in the solution phase is not seen because of its negligible magnitude. The figure shows that while the contact and matrix resistance are significant throughout the discharge, the overpotential becomes important at the end of discharge. The role of diffusion is not apparent in Figure 7 (a) and is masked by the overpotential drop.

In order to extract the effect of diffusion, a simulation was conducted with the same parameters as those used in 7(a), but with a diffusion coefficient in the solid phase three ordersof-magnitude larger. Considering the relative unimportance of the solution phase, the diffusion coefficient of the salt was left unchanged. The resulting plot, with the various drops listed, is shown in Figure 7 (b). Two curves in Figure 7 (b) differ from those in Figure 7 (a), namely the overall curve representing the voltage between the current collector and the reference electrode, 
and the curve representing the matrix potential drop. While the former change occurs because of a smaller diffusion overpotential and a larger utilization, the latter occurs because the larger $\mathrm{D}_{\mathrm{Li}}$ changes the reaction distribution in the porous electrode and hence changes the resulting drop. However, comparison of Figure 7 (a) and (b) shows that this change is small.

Figure 7 (b) allows separation of the kinetic drop from the transport drop via the difference between the electrode-potential curve and the one where the contact resistance and the matrix resistance are removed. This value is a small fraction of the total drop in the cell (about $40 \mathrm{mV}$ due to kinetics in an overall drop which ranges from 0.4 to $0.9 \mathrm{~V}$ depending on the SOD). Clearly a larger exchange current density would make this value even smaller. The drop due to transport is then evaluated by taking the difference between the electrode-potential curve in Figure 7 (a) and 7 (b). This allows us to combine and synthesize the two figures to give Figure 7 (c), which has the various drop's listed. It is clear that while the major portion of the voltage drops occur due to ohmic effects, diffusion significantly decreases the utilization of the cell. This is shown using the dotted line, which represents the curve one would have measured if the diffusion coefficient were large. For comparison, Figure 7 (c) also shows the potential drop in the liquid phase between the current collector and the separator end of the porous electrode. Although this drop does not add into the voltage drops listed in the figure, it serves to illustrate the relative unimportance of the liquid phase in determining the performance of the cell.

Figure 7 (c) suggests that, in order to improve the performance of the $\mathrm{LiFePO}_{4}$ electrode, both electron transport and ion transport have to be improved. The former can be achieved by improving the carbon coating in the current collector to eliminate the contact resistance, ${ }^{39}$ and by doping the material ${ }^{4}$ or adding more carbon to the cathode mix to increase the matrix-phase conductivity. The latter can be achieved by decreasing the particle size, and to a smaller extend, 
by having particles of uniform size in the electrode. Recent developments suggest that these strategies are indeed helpful in achieving better performance. ${ }^{45}$

\section{Design Considerations:}

The relative importance of each of these improvements is evaluated in Figure 8, which shows these effects in the form of a Ragone plot. Considering that this paper involves experimental and modeling studies on half cells against a Li metal negative electrode, we do not report the Ragone plot on a mass basis, but instead report them on an area basis. While this serves our purpose of estimating the relative importance of ohmic and transport effects adequately, it has the disadvantage that changes in cell design (thickness, porosity) cannot be explored, as such studies need to account for changes in the weight with change in design. This comprehensive study is performed in the subsequent paper where the design of a graphite/ $\mathrm{LiFePO}_{4}$ cell is optimized to maximize the specific energy. ${ }^{18}$

The energy per unit electrode area in Figure 8 was evaluated by finding the area under the voltage-capacity curve using $2.5 \mathrm{~V}$ as the cut-off potential. The power per unit electrode area was evaluated by dividing the energy by the time of discharge and hence represents an average value. The figure shows the experimental data and the model predictions for the cell shown in Figure 5, where the excellent fits seen in the discharge curve translates into similar fits for the energy. When the ohmic drops in the solid phase are eliminated, both in the contact and in the matrix, a significant improvement in the high-power performance of the cell is seen, owing to the higher voltage of the system which extends the capacity at which the potential of the electrode reaches the cut-off potential. However, little change is seen at low and intermediate rates because of the negligible effect of ohmic drops when the current is small. Note that it is assumed 
that both ohmic drops in the system can be eliminated without any penalty in the volume faction of the active material or the porosity of the system. One could think of this as a doped $\mathrm{LiFePO}_{4}$ electrode that has a much larger intrinsic conductivity.

When the particle sizes are made uniform at the average particle size (in additional to having no ohmic drops), a small increase in the performance of the cell is seen, especially at intermediates rates. As noted by Darling and Newman ${ }^{42}$ the performance of an electrode with a two-particle distribution is always worse than that where all the particles are at the average size because there are some particles that are larger than the average, which give rise to transport limitations. The low diffusion coefficient in the $\mathrm{LiFePO}_{4}$ electrode results in loss in utilization even at the small currents (see Figure 5), and hence the loss of the large particles results in an improvement in the capacity and thereby the energy of the cell at these rates. However, at higher currents, these differences become smaller, with the two curves merging. Under these conditions, the electrode reaches the cut-off potential far before the larger particles are completely accessed, and so the smaller particles become more important. As the smaller particle size is very close to the average particle size (a factor of 1.2 smaller), little change is seen in the cell behavior. However, Figure 8 suggests that if the distribution of particles were larger than those used in this study, the performance could be significantly different.

However, in practice, fabricating electrodes with a uniform particle size may not be feasible. So instead, the two-particle-size model was used with the average particle size made smaller to half its original value, with the ratio of the small and large particles to the average particle size maintained the same. Under these conditions a significant improvement in performance is seen at the higher currents. As much as a doubling of the power of the cell can be achieved when compared to the cell with the particle size used in Figure 5. The significant 
transport limitations in the electrode mean that the halving of the particle size will impact the utilization significantly. Note that making the particle size smaller with the porosity kept the same will result in a doubling of the surface area. However, as the kinetic resistance is small, this has only a small impact on the improved performance shown in Figure 8.

When the particle radius is made even smaller, to $13 \mathrm{~nm}$ radius, a further improvement in performance is seen, although the increase is not substantial. This improvement will continue with decrease in particle size until the liquid phase starts to dominate the behavior, and hence the performance, of the cell. Alternatively, when cells are made with negative electrodes that undergoes intercalation (e.g., graphite), transport in the negative particles may be the cause for the performance limitations, a problem which is nonexistent when using a Li-metal anode. In summary, Figure 8 suggests that while reducing contact resistances and increasing the matrix conductivity would help in improving the performance of the cell, a significant effect would arise from making the particle size smaller. However, an optimum particle size has other considerations that are not addressed in a mathematical model. For example, decreasing the particle size results in increased area, whereby any side reactions may start to become more prominent, resulting in less cycleable lithium. In addition, it may not be possible from a synthesis standpoint to make particles smaller than a particular length scale. Smaller particles may also be disadvantageous from a electrode fabrication standpoint. As pointed out by Chen and Dahn, ${ }^{46}$ smaller particle size electrodes would require the use of more carbon in order to coat each particle, resulting in a significant decrease in the tap density (the density of a packed powder wherein packing is achieved via tapping under standard conditions), which in turn may lead to a decrease in the volumetric energy density of the cell. Finally, smaller particles may be difficult to bind together in the form of an electrode and hence may result in greater ohmic drops 
because of poor contacts. These issues are difficult to address and hence a recommendation on the optimum particle size should be made with caution, although Figure 8 suggests that sizes smaller than $26 \mathrm{~nm}$ would not provide significant improvement in performance.

Finally, we use the model to address the issue of changing the electrolyte from a liquid to a gel system. For low-cost Li-ion cells, gel electrolytes offer a significant cost advantage compared to liquids because of the elimination of the expensive separator. Gel systems, however, suffer from a significantly smaller conductivity when compared to liquids. Doyle et al. measured the conductivity of gels, and in comparing them to the liquid systems, concluded that the conductivity was considerably lower than that predicted by the Bruggeman equation. ${ }^{9}$ The authors showed that while the Bruggeman equation predicts an $\varepsilon^{1.5}$ correction, an $\varepsilon^{4.5}$ dependence was needed. In addition, in calculating the effective properties in the porous media, the authors used an exponent of 3.3 (corresponding to a tortuosity of 2.9) as opposed to a value of 1.5. ${ }^{9}$ This correction factor was estimated based on comparison of a mathematical model (very similar to the one presented in this paper) and experimental data. While it is not clear whether this exponent of 3.3 is a property of the positive electrode, or if it is caused by the use of the gel electrolyte, the fact that the Bruggeman exponent breaks down even when there is no porous medium suggests that the gel plays a part in this deviation. The consequence of the larger exponent is an almost order of magnitude lower transport property in the porous medium compared to the free electrolyte for the low-porosity electrodes used in this study. In order to test the effect of having this lowered property in the $\mathrm{LiFePO}_{4}$ system, simulations were conducted at various rates using the properties measured by Doyle et al. on $\mathrm{LiPF}_{6}$ salts in a 1:2 $\mathrm{v} / \mathrm{v}$ mixture of EC/DMC in $\mathrm{p}(\mathrm{VdF}-\mathrm{HFP})$, a random copolymer of vinylidene fluoride and hexafluoropropylene. Gels have been used in $\mathrm{LiFePO}_{4}$-based $\mathrm{Li}$-ion cells recently, ${ }^{47}$ and hence 
these simulations are expected to be useful is providing information on the losses that occur under these conditions.

Figure 9 summarizes the results of these simulations in the form of a Ragone plot. The plot includes the experimental data in Figure 5 along with the model prediction. When the same cell is now simulated with a gel electrolyte, a significant decrease in the power capability is observed. Not only does the lower conductivity result in greater ohmic drop, but also the cells which operate at higher currents are limited by liquid-phase transport limitations which result in the electrolyte depletion in the interior of the porous electrode. Therefore, in these simulations, the limitation of the system shifts from the solid phase at low currents to the electrolyte phase at higher currents. Clearly the low porosity used in this study is not suitable for a gel electrolyte system, and considerable improvement may be possible by increasing the porosity of the material. No attempts are made in this paper to study this effect because increasing the porosity would result in a lower amount of active mass, and hence the weight of the electrode would be considerably different. However, the basis used in this study (per cross section area) does not allow us to address this mass issue adequately, and hence we leave this aspect unexplored.

However, one relatively straightforward way of circumventing this transport limitation in the system is to increase the salt concentration. Figure 9 shows simulations at a concentration of $2 \mathrm{M}$, where an improvement in performance is seen, although the liquid electrolyte still shows significantly higher performance. While the use of $2 \mathrm{M}$ salt concentration results in the concentration crossing the conductivity maximum, it allows the porous electrode to discharge longer before transport limitations take over. However, increasing the salt concentration can be detrimental from a cost standpoint, and higher concentrations may result in precipitation of the salt, especially near the negative electrode. 
In summary, Figure 9 asserts that using gel electrolytes would shift the limitations from the solid to the liquid phase, hence necessitating design changes (porosity, thickness) in order to make the performance comparable. In addition, increasing the matrix conductivity would shift the reaction to the separator end of the porous electrode, thereby reducing the depletion in the interior and resulting in better performance. However, considering that an optimum cell would be one where all the limitations in the system are comparable, a detailed study of the gel system may help in achieving acceptable performance while minimizing cost. Such a study will be the subject of future communications.

\section{Conclusion}

The $\mathrm{LiFePO}_{4}$ electrode has been known to have poor high power capability. We developed a mathematical model for this system in order to understand the cause for this poor performance. The phase change in the material is accounted for by assuming that the juxtaposition of the phases is in the form of a shrinking core. The diffusion of Li through the shell and the movement of the phase boundary are described mathematically and incorporated into a porous electrode model. Transport limitations leading to the inability to consume the core leads to loss in utilization in this material. While transport limitations are generally controlled by the particle size and the diffusion coefficient, we show that in phase-change systems the composition range of the single-phase region has a similar effect.

Half-cell experiments are performed to extract the equilibrium potential of the system as a function of SOD, which shows the existence of narrow single-phase regions between a SOD of 0 and 0.02 and 0.9525 and 1.0. These concentrations allow us to complete the description of the phase change in the material. Experimental discharge curves were then compared to model 
simulations where unknown parameters are extracted using the various features in the discharge curves. A two-particle size distribution was used to predict the utilization at various rates, while a contact resistance between the current collector and the porous electrode along with a low matrix conductivity was used to predict the voltage drops. Simulations show that in addition to these ohmic effects, solid-phase diffusion limitations also contribute to the low-power capability of the material. While the resistances are significant all through the discharge, the diffusion limitations starts to dominate at the end of discharge. The relative importance of each of these losses was investigated by using Ragone plots. Decreasing ohmic effects and decreasing the particle size were both seen to be very critical. Although the benefits of having a uniform particle size were seen to be less significant, this may be more important if the distribution were wide. Particle radii less than $26 \mathrm{~nm}$ show little improvement in performance, suggesting a possible design criterion. Operating the electrode with a gel electrolyte instead of a liquid changes the limitation from the solid phase to the electrolyte phase, thereby decreasing the power capability of the material. Making electrodes with larger porosity than ones used in this paper would be a prerequisite for this type of cell design.

More information on the juxtaposition of the two phases would be critical in making the model complete. The validity of using a symmetric shrinking core and the assumption that grain-boundary diffusion does not play any part in transport need to be reexamined. A spectroscopic confirmation of the range of the single-phase region, possibly by lithiating to a limited extend at the two SOD extremes and performing XRD experiments, would eliminate any artifacts that open-circuit measurements may be subject to. Finally, a better understanding of electron conduction in the material, especially any changes in conductivity on $\mathrm{Li}$ insertion/extraction in the single-phase regions would help in refining the model. 


\section{Appendix A}

This appendix details the behavior of a single particle of active material which undergoes a phase change via a shrinking core in order to understand how the behavior differs from that of an intercalation electrode and to identify the controlling parameters. We accomplish these two tasks using dimensionless equations.

Equations 2 to 5 can be recast into a dimensionless form by making the concentration dimensionless with the maximum concentration $\left(\theta=\frac{\mathrm{c}_{\mathrm{s}}}{\mathrm{c}_{\max }}\right)$, the radial coordinate with the radius of the particle $\left(\xi=\frac{\mathrm{r}}{\mathrm{r}_{\mathrm{o}}}\right.$ and $\left.\xi_{\mathrm{i}}(\mathrm{t})=\frac{\mathrm{r}_{\mathrm{i}}(\mathrm{t})}{\mathrm{r}_{\mathrm{o}}}\right)$, and time with the diffusion coefficient and the square of the radius $\left(\tau=\frac{t_{\mathrm{Li}}}{\mathrm{r}_{\mathrm{o}}^{2}}\right)$. Assuming that the initial concentration is zero $\left(\mathrm{c}_{\mathrm{o}}=0\right)$, these equations yields two parameters, namely $I^{*}=\frac{i_{n} r_{0}}{D_{L i} c_{\max } F}$ and $\delta=\frac{c_{e q}}{c_{\max }}$. The first is a dimensionless current, and the second quantifies the composition range of the single-phase region. The resulting equations are

$$
\begin{aligned}
\frac{\partial \theta}{\partial \tau}=\frac{\partial^{2} \theta}{\partial \xi^{2}}+\frac{2}{\xi} \frac{\partial \theta}{\partial \xi} & \\
\theta=0 & \text { at } \tau=0 \\
\frac{\partial \theta}{\partial \xi}=I^{*} & \text { at } \xi=1 \\
\theta=\delta & \text { at } \xi=\xi_{\mathrm{i}}(\mathrm{t})
\end{aligned}
$$

with the movement of the phase interface given by 


$$
-\delta \frac{\mathrm{d} \xi_{\mathrm{i}}}{\mathrm{d} \tau}=\left.\frac{\partial \theta}{\partial \xi}\right|_{\xi=\xi_{\mathrm{i}}}
$$

When the core shrinks to the center of the particle the boundary condition at $\xi=\xi_{\mathrm{i}}(\mathrm{t})$ is changed to

$$
\frac{\partial \theta}{\partial \xi}=0 \quad \text { at } \xi=0
$$

For an intercalation electrode, $=0$ and no core exists and hence Equation A4 is used at all times.

Figure A-1 illustrates the shrinking core and the change in boundary conditions involved in the single-particle model. The graph shows the concentration profiles in a single particle at various times at a dimensionless current, $\mathrm{I}^{*}$, of 0.03 and for $\delta=0.9$ (equivalent to a $10 \%$ width for the single-phase region). As the phase interface is maintained at a constant concentration, the inner boundary remains at a value of 0.9 until the core is consumed. The flux at this boundary drives the movement of the two-phase interface. As discharge proceeds, the core shrinks until it reaches the center of the particle. The boundary conditions are then shifted to Equation A4 (symmetry at the center of the particle), which is seen in the line corresponding to a $\tau \mathrm{I}^{*}$ of 0.33 . However, note that the amount of active material in this single-phase region is small, as shown by the dimensionless concentration being close to 1.0 in the figure. The end of discharge occurs when the surface concentration reaches the maximum concentration $(1.0$ due to the normalization scheme used). In Figure A-1, this occurs after the core is consumed.

However, this consumption of the core depends on the both $\mathrm{I}^{*}$ and $\delta$, as illustrated in Figure A-2, which shows both the utilization and the dimensionless radius of the core at the end of discharge (when the dimensionless surface concentration reaches 1.0) for various values of I*. 
Here the utilization is defined as $3 \tau \mathrm{I}^{*}$, where the 3 arises from the ratio of the area to the volume in a spherical particle. The graph shows these quantities for three values of $\delta$, namely $0.99,0.9$ (both corresponding to a phase-change electrode) and 0 (corresponding to an intercalation electrode). For the intercalation electrode, there is no core radius, and hence the quantity is not plotted.

For the phase-change electrode, at small values of $\mathrm{I}^{*}$ the core is completely consumed by the end-of-discharge (dimensionless radius of the core=0), and the utilization is large. As the current increases, less of the core is consumed, and correspondingly the utilization decreases. At very high currents, the core remains at the surface of the particle, and the electrode does not provide any useable capacity. In other words, the utilization of the particle is large as long as the core shrinks significantly.

In addition to $\mathrm{I}^{*}$, the width of the single-phase region also plays a part in deciding the utilization of the cell, also illustrated in Figure A-2. As the value of $\delta$ increases, the current at which the utilization decreases starts to decrease. In other words, the narrower the single-phase region, the smaller the dimensionless current will be where the electrode will provide useable capacity. Figure A-2 shows the usefulness of using an intercalation electrode for battery applications, where the wide concentration range allows for larger currents to be used, thereby providing a significant advantage over a phase-change electrode. In summary, Figure A-2 suggests that in order for a phase-change electrode to be useful for high-power applications (i.e., sustain large currents), one would need to use small particles, make materials that have a larger diffusion coefficient, or extend the width of the single-phase region and the end of discharge. 


\section{Appendix B}

This appendix lists the electrolyte properties used in this paper. For the liquid system the diffusion coefficient of the salt was estimated at a particular concentration using

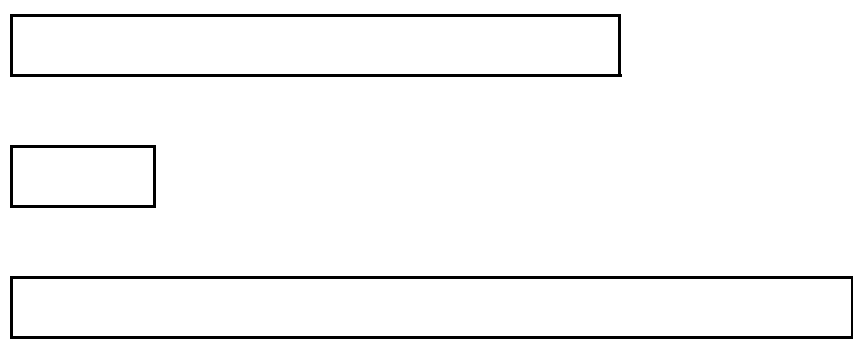

where $\mathrm{c}$ is the salt concentration in mole/liter, D the diffusion coefficient, $\square$ the transference number and $\kappa$ the conductivity. While the diffusion coefficient and transference number were taken from reference 48 and correspond to LiPF6 in EC:EMC, the conductivity was taken based on reference 33 .

For the gel simulations, the following parameters were taken from reference 9

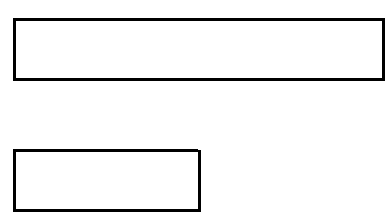




\section{Acknowledgements}

Financial support from the Assistant Secretary for Energy Efficiency and Renewable Energy, Office of FreedomCAR and Vehicle Technologies of the U.S. Department of Energy under Contract No. DE-AC03-76SF00098 is gratefully acknowledged. We acknowledge Kathy Striebel and Joongpyo Shim (LBNL) for providing the equipment and electrodes used in this study, help with the experimentation, and for discussions. William H. Tiedemann (Johnson Controls, retired) is acknowledged for comments and suggestions during the preparation of this manuscript. 


\section{Notation}

$c_{\mathrm{eq}} \quad$ concentration of lithium in the shell at the phase-interface, $\mathrm{mol} / \mathrm{m}^{3}$

$\mathrm{c}_{\max }$ maximum concentration of lithium in the iron-phosphate lattice, $\mathrm{mol} / \mathrm{m}^{3}$

$\mathrm{c}_{\mathrm{o}} \quad$ initial concentration of lithium in the iron-phosphate lattice, $\mathrm{mol} / \mathrm{m}^{3}$

$\mathrm{c}_{\mathrm{s}} \quad$ concentration of lithium in the iron-phosphate lattice, $\mathrm{mol} / \mathrm{m}^{3}$

D diffusion coefficient of the salt in the electrolyte phase, $\mathrm{m}^{2} / \mathrm{s}$

$\mathrm{D}_{\mathrm{Li}}$ diffusion coefficient of lithium in the iron-phosphate lattice, $\mathrm{m}^{2} / \mathrm{s}$

$\mathrm{D}_{0}, \mathrm{D}_{1}$ ranges used in the concentration-dependent diffusion coefficient equation (Equation 12), $\mathrm{m}^{2} / \mathrm{s}$

F $\quad$ Faradays constant, $96487 \mathrm{C} / \mathrm{eq}$

$I^{*} \quad$ dimensionless current

$\mathrm{i}_{\mathrm{n}} \quad$ reaction current, $\mathrm{A} / \mathrm{m}^{2}$

$\mathrm{i}_{\mathrm{o}} \quad$ exchange current density, $\mathrm{A} / \mathrm{m}^{2}$

$\mathrm{n} \quad$ parameter in Equation 12

$\mathrm{r} \quad$ radial coordinate, $\mathrm{m}$

$r_{i} \quad$ radius of the phase interface in the iron-phosphate particle, $m$

$r_{o} \quad$ radius of the iron-phosphate particle, $m$

$\mathrm{t}$ time, $\mathrm{s}$

transference number of lithium in the electrolyte 
$\mathrm{U}$ equilibrium potential of the iron-phosphate electrode, $\mathrm{V}$

$\mathrm{x}$ dimensionless coordinate used to transform the moving boundary

\section{Greek}

$\delta$ dimensionless concentration of lithium in the shell at the phase-interface

$\kappa \quad$ conductivity of the electrolyte phase, $\mathrm{S} / \mathrm{m}$

$\tau \quad$ dimensionless time

$\theta$ dimensionless concentration of lithium in the iron-phosphate lattice

$\xi \quad$ dimensionless radial coordinate

$\xi_{\mathrm{i}} \quad$ dimensionless radius of the phase-interface in the iron-phosphate particle 


\section{References}

1. A. K. Padhi, K. S. Nanjundaswamy, and J. B. Goodenough, J. Electrochem. Soc., 144, 1188 (1997).

2. J. B. Goodenough, A. K. Padhi, K. S. Nanjundaswamy, and C. Masquelier, US Patent $5,910,382(1999)$.

3. A. Yamada, S. C. Chung, and K. Hinokuma, J. Electrochem. Soc., 148, A224 (2001).

4. S.-Y. Chung, J. T. Bloking, and Y.-M, Chiang, Nature Mat., 2, 123 (2002).

5. N. Ravat, J. B. Goodenough, S. Besner, M. Simoneau, P. Hovington, and M. Armand, Abstract 127, The Electrochemical Society and The Electrochemical Society of Japan Meeting Abstracts, Vol 99-2, Honolulu, HI (1999).

6. H. Huang, S.-C., Yin, and L. F. Nazar, J. Electrochem. Soc., 4, A170 (2001).

7. K. West, T. Jacobsen, and S. Atlung, J. Electrochem. Soc., 129, 1480 (1982).

8. M. Doyle, T. F. Fuller, and J. Newman, J. Electrochem. Soc., 140, 1526 (1993).

9. M. Doyle, J. Newman, A. S. Gozdz, C. N. Schmutz, and J.-M. Tarascon, J. Electrochem.Soc., 143, 1890 (1996).

10. P. Arora, M. Doyle, A. S. Gozdz, R. E. White, and J. Newman, J. Power Sources, 88, 219 (2000).

11. G. S. Nagarajan, J. W. Van Zee, and R. M. Spotnitz, J. Electrochem. Soc., 145, 771 (1998).

12. M. W. Verbrugge and B. J. Koch, J. Electrochem. Soc., 150, A374 (2003).

13. M. Doyle and Y. Fuentes, J. Electrochem. Soc., 150, A706 (2003). 
14. R. Darling, J. Newman, J. Electrochem. Soc., 145, 990 (1998).

15. P. Arora, M. Doyle and R. E. White, J. Electrochem. Soc., 146, 3553 (1999).

16. A. Yamada, Y. Kudo, and K. -Y, Liu, J. Electrochem. Soc., 148, A1153 (2001).

17. R. Pollard and J. Newman, J. Electrochem. Soc., 128, 491 (1981).

18. V. Srinivasan and J. Newman, Optimization of a natural-graphite/iron phosphate Li-ion cell, J. Electrochem. Soc., Submitted (2003).

19. K. E. Thomas, R. M. Darling, and J. Newman, in Advances in Lithium-Ion Batteries, W. van Schalkwijk and B. Scrosati, ed., New York: Kluwer Academic/Plenum Publishers, 345 (2002).

20. J. Newman and W. Tiedemann, J. Electrochem. Soc., 144, 3081 (1997).

21. Y. Q. Lei, C. S. Wang, X. G. Yang, H. G. Pan, J. Wu, and Q. D. Wang, J. Alloys \& Comp., 231, $611(1995)$.

22. W. Zhang, S. Srinivasan, and H. J. Ploehn, J. Electrochem. Soc., 143, 4039 (1996).

23. N. Cui, J. L. Luo, and K. T. Chuang, J. Electroanal. Chem., 503, 92 (2001).

24. V. R. Subramanian, H. J. Ploehn, and R. E. White, J. Electrochem. Soc., 147, 2868 (2000).

25. R. Pollard, Mathematical Modeling of the Lithium-Aluminum, Iron Sulfide Battery, , PhD. Dissertation, University of California, Berkeley (1979).

26. J. S. Newman, Electrochemical Systems, Englewood Cliffs, NJ (1991).

27. J. Newman, Electroanalytical Chemistry, Vol. 6, A. J. Bard, Editor, Marcel Dekker, Inc., New York (1973). 
28. R. White, J. A. Trainham, J. Newman and T. W. Chapman, J. Electrochem. Soc., 124, 669 (1977).

29. J. Newman, I\&EC Fundamentals, 7, 514 (1968).

30. B. Paxton and J. Newman, J. Electrochem. Soc., 144, 3818 (1997).

31. C. J. Wen, B. A. Boukamp, R. A. Huggins, and W. Weppner, J. Electrochem. Soc., 126, 2258 (1979).

32. T. W. Farrell, C. P. Please, D. L. S. McElwain, and D. A. J. Swinkels, J. Electrochem. Soc., 147, $4034(2000)$

33. C. M. Doyle, Design and Simulation of Lithium Rechargeable Batteries, PhD. Dissertation, University of California, Berkeley (1995).

34. P. P. Prosini, M. Lisi, D. Zane, and M. Pasquali, Solid State Ionics, 148, 45 (2002).

35. P. P. Prosini, M. Lisi, S. Scaccia, M. Carewska, F. Cardellini, and M. Pasquali, J. Electrochem. Soc., 149, A297 (2002).

36. S. Franger, F. L. Cras, C. Bourbon, and H. Rouault, Elec. Solid State Lett., 5, A231 (2002).

37. M. M. Doeff, Y. Hu, F. McLarnon, and R. Kostecki, Elec. Solid State Lett., 6, A207 (2003).

38. P. Arora, M. Doyle, A. S. Gozdz, R. E. White and J. Newman, J. Power Sources, 88, 219 (2000).

39. Batteries for Advanced Transportation Technologies quarterly review, http://eande.lbl.gov/BERC/BATT/BATT.html , pg. 5, April 2004.

40. B. Paxton and J. Newman, J. Electrochem. Soc., 143, 1287 (1996). 
41. S. Motupally, C. C. Streinz, and J. W. Weidner, J. Electrochem. Soc., 145, 29 (1998).

42. R. Darling and J. Newman, J. Electrochem. Soc., 144, 4201 (1997).

43. G. S. Nagarajan, J. W. Van Zee, and R. M. Spotnitz, J. Electrochem. Soc., 145, 771 (1998).

44. J. Weidner and P. Timmerman, J. Electrochem. Soc., 141, 346 (1994).

45. K. Striebel, J. Shim, V. Srinivasan, and J. Newman, Abstract 348, The Electrochemical Society Extended Abstracts, Orlando, Fl, Oct 12-16, 2003.

46 Z. Chen and J. R. Dahn, J. Electrochem. Soc., 149, A1184 (2002).

47. K. Striebel, A. Guerfi, J. Shim, M. Armand, M. Gauthier, and K. Zaghib, J. Power Sources, 119, 951 (2003).

48. C. Capiglia, Y. Saito, H. Kageyama, P. Mustarelli, T. Iwamoto, T. Tabuchi, and H. Tukamoto, J. Power Sources, 81-82, 859 (1999). 
Table 1. List of parameters used to make the plots in this paper. The table shows both the design-adjustable parameters and those that are electrode specific. In Figure $8, r_{\text {avg }}$ was decreased while keeping the ratio of small and large particles to the average particle size the same.

\begin{tabular}{|c|c|}
\hline Parameter & Value \\
\hline \multicolumn{2}{|c|}{ Design-adjustable Parameters } \\
\hline Electrode thickness & $62 \mu \mathrm{m}^{\mathrm{a}}$ \\
\hline Porosity & $0.25^{\mathrm{b}}$ \\
\hline Volume fraction of active material & $0.3407^{\mathrm{b}}$ \\
\hline Initial salt concentration & $1 \mathrm{M}^{\mathrm{a}}$ \\
\hline Separator thickness & $25 \mu \mathrm{m}^{\mathrm{a}}$ \\
\hline Average particle radius $\left(r_{0}\right)$-base case & $52 \mathrm{~nm}^{\mathrm{b}}$ \\
\hline Small particle radius & $\mathrm{r}_{\mathrm{o}} / 1.2^{\mathrm{c}}$ \\
\hline Large particle radius & $\mathrm{r}_{0} \times 1.8^{\mathrm{c}}$ \\
\hline \multicolumn{2}{|l|}{ Electrode Parameters } \\
\hline Diffusion coefficient in $\mathrm{LiFePO}_{4}\left(\mathrm{D}_{\mathrm{Li}}\right)$ & $8 \times 10^{-18} \mathrm{~m}^{2} / \mathrm{s}^{\mathrm{c}}$ \\
\hline Exchange current density (reference concentration $1 \mathrm{M}$ and & $3.14 \times 10^{-6} \mathrm{~A} / \mathrm{m}^{2 \mathrm{c}}$ \\
\hline \multicolumn{2}{|l|}{$50 \% \mathrm{SOC})$} \\
\hline Matrix phase conductivity & $5 \times 10^{-3} \mathrm{~S} / \mathrm{m}^{\mathrm{c}}$ \\
\hline Contact resistance & $0.0065 \Omega \mathrm{m}^{2 \mathrm{c}}$ \\
\hline
\end{tabular}

\footnotetext{
${ }^{\text {a }}$ Measured

${ }^{\mathrm{b}}$ Estimated

${ }^{c}$ Extracted from model-experimental comparison as described in the text. See section: Model-Experimental Comparison.
} 

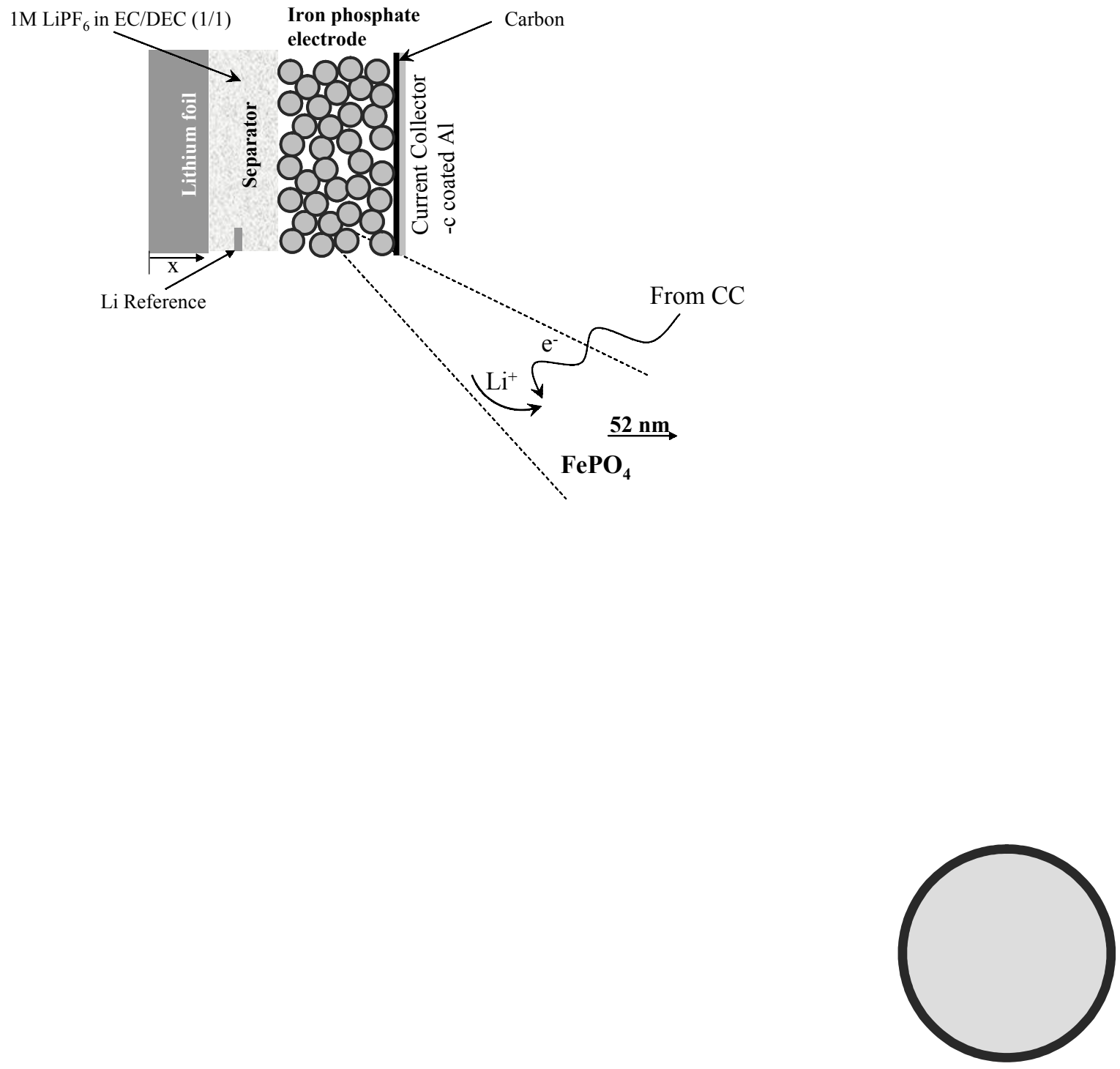

Figure 1. Schematic of the cell modeled in the study, consisting of a Li-metal negative and $\mathrm{LiFePO}_{4}$ positive with a separator between them. The cell is filled with electrolyte. The $\mathrm{LiFePO}_{4}$ porous electrode is attached to a carbon-coated aluminum current collector. The electrode is assumed to consist of spherical particles at the surface of which electrochemical reaction occurs with the $\mathrm{Li}^{+}$ion reaching the reaction site from the solution and the $\mathrm{e}^{-}$traveling through the carbon backbone from the current collector. 

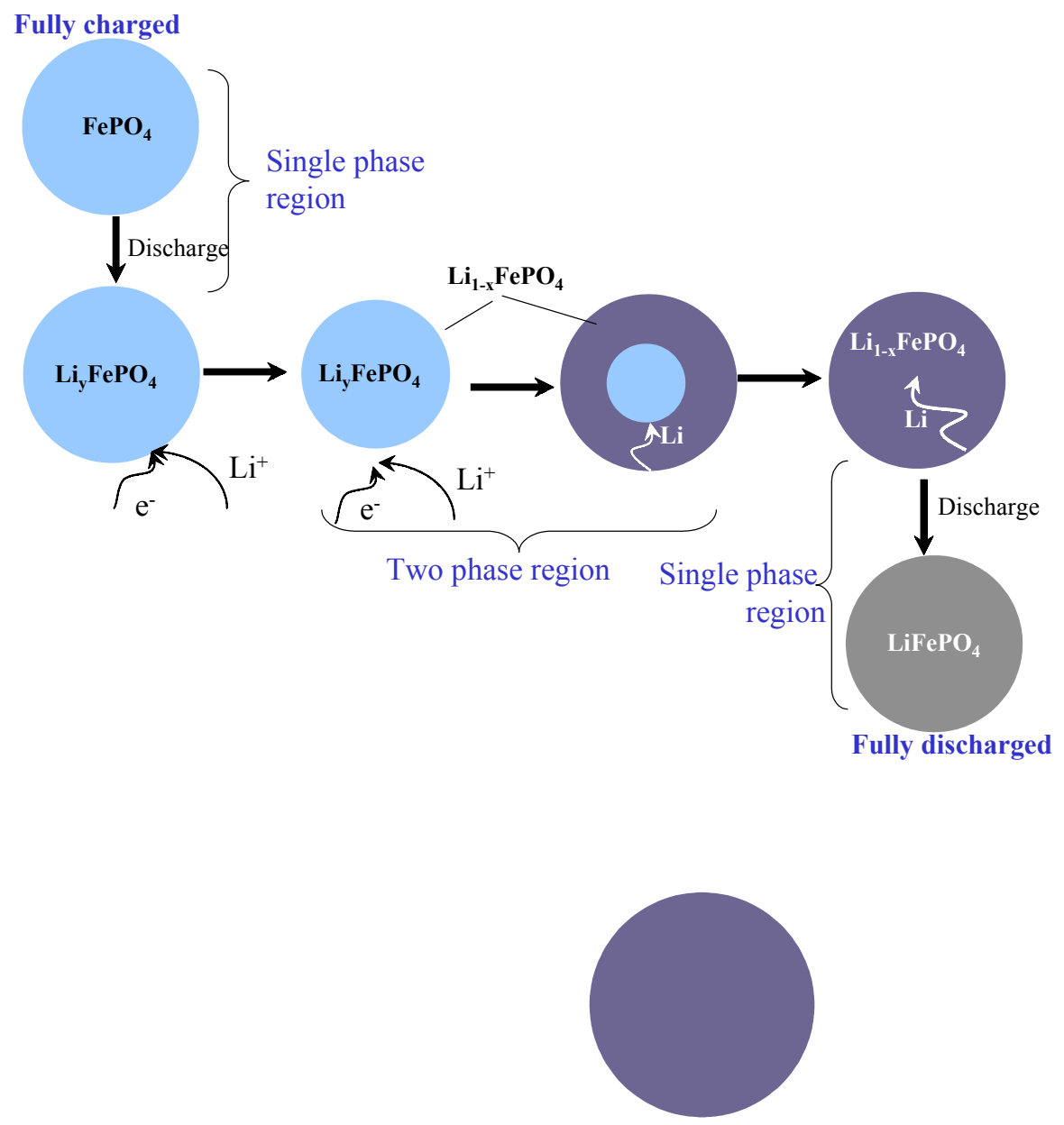

Figure 2. Illustration of the shrinking-core model with the juxtaposition of the two phases and the movement of the phase boundary. The processes during discharge are illustrated. Both the single-phase and the two-phase regions are shown. See text for details. 
Figure 3. Open-circuit potential of the iron-phosphate electrode as a function of the state-of-discharge $\left(\mathrm{c} / \mathrm{c}_{\max }\right)$. The symbols denote the data which were taken by discharging to a particular SOD and the resting the cell to reach a steady state. The data show the two narrow single-phase regions, the two-phase plateau, and the concentration ranges that separate them. The line denotes the fit to the data using Equation 11 which was used in the mathematical model. 
(a)

(b)

Figure 4. Constant-current density discharge curves of the iron-phosphate electrode at different current densities (4a) and model predictions using a particle radius of $52 \mathrm{~nm}$ (4b). The figure shows the significant drop in both the utilization and the operating voltage as current is increased. The model fits show the inability of the model to predict the utilization at all currents when using a single particle size. 
Figure 5. Model experimental comparisons at various rates showing the ability of the model to describe accurately the different features. The methodology used to obtain the fit is detailed in the text. This figure was generated using a two-particle model in order to fit the utilization at various rates. Compare with Figure 4, where a single-particle model was used. 
(a)

(b)

Figure 6. Pore-wall flux across the $\mathrm{LiFePO}_{4}$ electrode at different points during the discharge for the large (5a) and small (5b) particles for the discharge at $2.6 \mathrm{~mA} / \mathrm{cm}^{2}$. The left side of the abscissa represents the separator, and the right side the current collector. The profiles show the movement of the reaction from the current collector into the interior as discharge proceeds. 
(a)

(b)

(c)

Figure 7. Estimation of the potential drops in the iron-phosphate electrode at a current density of $2.6 \mathrm{~mA} / \mathrm{cm}^{2}$. The graph shows the contributions that result in the potential deviating from that of the open-circuit. Figure 7(a) was made using the values reported in Figure 5 and 7(b) using a large diffusion coefficient in the solid phase. The overall qualitative drops are shown in 7 (c). See text for details on the methodology for determining the drops. 
Figure 8. Ragone plot showing the effect of decreasing the ohmic and transport losses in the iron-phosphate electrode. The graph shows the data and the model fits from Figure 5 and four other lines, all of which have no matrix resistance or contact resistance. All lines, except the one marked "single particle," use the two particle model. 'Present cell' represents the simulation using the parameters in Table 1. 
Figure 9. Ragone plot showing the effect of changing the system from a liquid electrolyte to a gel electrolyte. The graph shows the experiments and the model simulations from Figure 5 in additional to gel cells with two different salt concentrations. All the simulations were conducted using values listed in Table 1. 
Figure A-1. Concentration profiles in a single spherical particle of $\mathrm{LiFePO}_{4}$ at a constant discharging current density, $I^{*}=0.03$ and $\delta=0.9$. The graph shows the evolution at various times and captures the shrinking of the core and the change in boundary condition when the core reaches the center of the particle. 
Figure A-2. The utilization and dimensionless core radius when the dimensionless surface concentration reaches 1.0 plotted at various dimensionless current densities for various values of $\delta$. The plot shows the lowered utilization at higher currents and the corresponding inability to shrink the core completely. The $\delta=0$ simulations are for an intercalation electrode, and hence no core radius exists. 